(W)

Check for

Cite as

Nano-Micro Lett.

(2021) 13:105

Received: 23 December 2020

Accepted: 9 February 2021

Published online: 5 April 2021

(C) The Author(s) 2021

\section{Pancake-Like MOF Solid-State Electrolytes with Fast Ion Migration for High-Performance Sodium Battery}

Gang Zhang ${ }^{1}$, Jun Shu ${ }^{1}$, Lin Xu ${ }^{1,2}{ }^{凶}$, Xinyin Cai ${ }^{1}$, Wenyuan Zou ${ }^{1}$, Lulu Du ${ }^{1}$, Song Hu ${ }^{1}$, Liqiang Mai ${ }^{1,2} \bowtie$

\title{
HIGHLIGHTS
}

- A pancake-like morphology solid-like eletrolyte of sodium battery with high ionic conductivity $\left(6.60 \times 10^{-4} \mathrm{~S} \mathrm{~cm}^{-1}\right)$ was obtained by simple hydrothermal method.

- Solid-like electrolyte with pancake-like morphology showed good interface contact and excellent compatibility (stable cycle over $500 \mathrm{~h}$ at $0.6 \mathrm{~mA} \mathrm{~cm}^{-2}$ ) with sodium metal.

- Provides possible repulsive force explanation for the restriction of ion transport by MOF.

\begin{abstract}
Solid-state electrolyte (SSE) of the sodium-ion battery have attracted tremendous attention in the next generation energy storage materials on account of their wide electrochemical window and thermal stability. However, the high interfacial impedance, low ion transference number and complex preparation process restrict the application of SSE. Herein, inspired by the excellent sieving function and high specific surface area of red blood cells, we obtained a solid-like electrolyte (SLE) based on the combination of the pancake-like metal-organic framework (MOF) with liquid electrolyte, possessing a high ionic conductivity of $6.60 \times 10^{-4} \mathrm{~S} \mathrm{~cm}^{-1}$, and excellent sodium metal compatibility. In addition, we investigated the
\end{abstract}

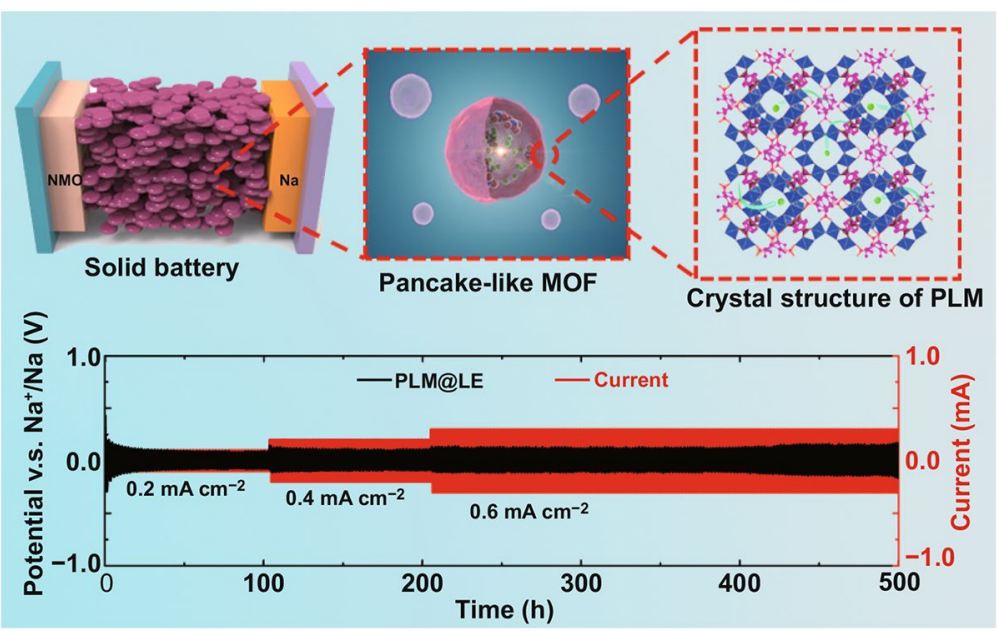
ion restriction effect of MOF's apertures size and special functional groups, and the ion transference number increased from 0.16 to 0.33 . Finally, the assembled $\mathrm{Na}_{0.44} \mathrm{MnO}_{2} / / \mathrm{SLE} / / \mathrm{Na}$ full batteries showed no obvious capacity decrease after 160 cycles. This material design of SLE in our work is an important key to obtain fast ion migration SLE for high-performance sodium-ion batteries.

KEYWORDS Metal-organic Frameworks; Sodium-ion Battery; Solid-like Electrolyte; Interface Contact

Lin Xu, linxu@whut.edu.cn; Liqiang Mai, mlq518@whut.edu.cn

1 State Key Laboratory of Advanced Technology for Materials Synthesis and Processing, School of Materials Science and Engineering, Wuhan University of Technology, Wuhan 430070, People's Republic of China

2 Foshan Xianhu Laboratory of the Advanced Energy Science and Technology Guangdong Laboratory, Xianhu Hydrogen Valley, Foshan 528200, People's Republic of China 


\section{Introduction}

With the development of science and technology, energy storage systems play an important role in portable equipment, transportation and electrostatic energy storage [1, 2]. As a potential energy storage system, sodium-ion batteries (SIBs) with rich sodium resources can meet most of the daily energy storage requirements [3-6]. However, traditional commercial liquid organic electrolytes in SIBs are likely to cause safety problems due to the flammability and leakage of electrolyte [7, 8]. Compared with liquid electrolyte (LE), solid-state electrolytes (SSEs) have a higher safety due to their high mechanical strength and wide voltage windows [9-12]. Nevertheless, SSEs have suffered some serious problems such as high interfacial impedance, low ion transference number and complex preparation process. Several methods were proposed to deal with the challenges of SSEs, but it is still far from the requirements of high-performance SSEs. For example, Gerbaldi et al. fabricated a polymer electrolyte with a single ionic conductor, increasing the ion transference number by sacrificing ionic conductivity [13]. Goodenough et al. improved the interface contact between the $\mathrm{Na}_{3} \mathrm{~V}_{2}\left(\mathrm{PO}_{4}\right)_{3}$ and the $\mathrm{Na}_{3} \mathrm{Zr}_{2}\left(\mathrm{Si}_{2} \mathrm{PO}_{12}\right)$ electrolyte by adding succinitrile, but this plastic-crystalline electrolyte was not very stable to sodium metals [14]. Hence, it is crucial to exploit a kind of novel SSEs with low interfacial impedance and high ion transference number.

On the one hand, high interfacial impedance results from poor contact between SSEs and electrodes [15]. This interface gap contact limits the overall sodium ion transport. Therefore, it is more advantageous to find a kind of SSEs that can quickly transfer sodium ions in the bulk phase as liquid electrolyte. Recently, metal-organic framework (MOF) has been widely used in many fields for its high specific surface area, permanent pore, adjustable function and simple preparation process [16-20]. Numerous works have focused on the use of MOF in the field of ion battery separator and solid-like electrolyte (SLE), where MOF's own large "cage" electrical insulation can be used to store LE and sieve different ions [21-24]. For example, Wang et al. proposed an interface wetting effect in lithium-ion batteries by compounding MOF with ionic liquid, which significantly improved the electrolyte/electrode interfacial impedance [21]. However, it is a pity that ionic liquid is easy to react with sodium metal, and the application of MOF in sodium ion SSEs is still very scarce. Furthermore, the MOF with appropriate pore size and high specific area has more advantages in electrochemical performance [25]. On the other hand, it is well known that red blood cells allow only some of the beneficial small molecules to pass through, which will encourage the design of SLE and hopefully increase ion transference number [26, 27]. Due to their pancake-like morphology, red blood cells have a higher specific surface area that allows them to transport more electrolytes and oxygen [28, 29]. In addition, the structural stability of the pancake-like morphology of red blood cells in human body is conducive to the design of SLE with good liquid retention and high safety to a certain extent. Considering the above reasons, it is an effective strategy to select a MOF with pancakelike morphologies to optimize ion transport and interfacial impedance in sodium-ion SLE.

Herein, pancake-like MIL-125 (PLM) with shape similar to red blood cells was prepared by a simple hydrothermal method. Its unique three-dimensional pore structure can be used as a "host" for LE and restrict the migration of anions (PLM@LE). Sodium ions can be transported quickly in the PLM due to the large size, high pore ratio and smooth pancake-like morphology, making SLE exhibit a high ionic conductivity of $6.60 \times 10^{-4} \mathrm{~S} \mathrm{~cm}^{-1}$ at room temperature and low activation energy of $0.112 \mathrm{eV}$. Meanwhile, unique aperture sizes and nucleophilic functional groups of PLM can limit the transport of anions, increasing the sodium ion transference number from 0.16 to 0.33 . Moreover, the LE inside the MOF can wet the point-to-point contact of MOF particles on the atomic level. This special wetting effect and good contact between PLM particles reduces the migration barrier of sodium ion, resulting in good compatibility between PLM@ LE and sodium metal. Finally, the assembled $\mathrm{Na}_{0.44} \mathrm{MnO}_{2} / /$ PLM@LE//Na SIBs can stable cycle for 160 cycles at $100 \mathrm{~mA} \mathrm{~g}^{-1}$ without obvious capacity degradation.

\section{Experimental Section}

\subsection{Materials Synthesis}

p-Phthalic acid (PTA 99\%), 1-Methyl-2-pyrrolidinone (NMP 99.9\%), 2-amino-terephthalic acid (98\%) were 
purchased from Aladdin. Titanium isopropoxide (97\%) was purchased from Macklin. $N, N$-Dimethylformamidel (DMF 99.5\%), Methanol (99.5\%), Isopropanol (99.7\% IPA) were purchased from SCR. $\mathrm{NaClO}_{4}$-PC with $5 \%$ FEC was purchased from DuoDuo. The reagents obtained were not further purified.

Synthesis of PLM: Typically, 3.0 g PTA was added to the mixture of $54 \mathrm{~mL} \mathrm{DMF}$ and $6 \mathrm{~mL}$ methanol, stirring continuously until the solution was transparent. Then slowly drop $1.56 \mathrm{~mL}$ titanium isopropoxide and transfer it into the reactor for hydrothermal reaction at $150{ }^{\circ} \mathrm{C}$ for $24 \mathrm{~h}$. The obtained material was centrifugally washed with DMF and methanol three times, and placed in a $70{ }^{\circ} \mathrm{C}$ vacuum oven overnight. The dried powder was activated in the vacuum at $120^{\circ} \mathrm{C}$ and stored in the argon glove box (water, oxygen $<0.1 \mathrm{ppm}$ ) waiting for use.

Synthesis of decanedron-like MIL-125 (DLM): $0.45 \mathrm{~mL}$ titanium isopropoxide and $0.747 \mathrm{~g}$ PTA were dissolved in the mixture of $13.5 \mathrm{~mL}$ DMF and $1.5 \mathrm{~mL}$ methanol. The subsequent hydrothermal time and temperature, as well as washing sample operation are consistent with PLM.

Synthesis of $\mathrm{NH}_{2}$-MIL-125: $0.45 \mathrm{~mL}$ titanium isopropoxide and $0.816 \mathrm{~g}$ 2-amino-terephthalic acid were dissolved in the mixture of $11.25 \mathrm{~mL}$ DMF and $3.75 \mathrm{~mL}$ methanol. The subsequent hydrothermal time and temperature, as well as washing sample operation are consistent with PLM.

Preparation of PLM@LE: Typically, a certain amount of activated PLM was compounded with different contents of $\mathrm{NaClO}_{4}$-PC with 5\% FEC electrolyte, and placed at $40{ }^{\circ} \mathrm{C}$ of heating platform for $6 \mathrm{~h}$.

Preparation of PLM@LE flexible membrane: PLM powder and polytetrafluoroethylene (PTFE) were ground evenly in IPA with a mass ratio of 9:1 by hand grinding. The obtained slurry was rolled into a thin film and cut into a small self-standing wafer with a thickness of about $120 \mu \mathrm{m}$. After that, PLM flexible film was placed in a $60{ }^{\circ} \mathrm{C}$ vacuum oven overnight to remove the excess IPA. A few drops of LE were added to the dried flexible membrane during the assembly of the battery.

Preparation of cathode: $\mathrm{Na}_{0.44} \mathrm{MnO}_{2}$ active material was synthesized according to the literature [30]. The working electrode was made by mixing $\mathrm{Na}_{0.44} \mathrm{MnO}_{2}$ (70 wt \%), PLM@LE (10 wt $\%)$, acetylene black (10 wt $\%$ ) and polytetrafluoroethyle (PVDF $10 \mathrm{wt} \%$ ) in NMP to get the uniform slurry. The slurry was coated on the $\mathrm{Al}$ foil and dried on a $70{ }^{\circ} \mathrm{C}$ coating machine for $12 \mathrm{~h}$. The electrode was cut into slices with the mass loading of $0.8-1.2 \mathrm{mg} \mathrm{cm}^{-2}$.

\subsection{Materials Characterization}

Crystal structure information of sample was conducted by D8 Discover X-ray diffractometer (XRD) using $\mathrm{Cu}-\mathrm{K} \alpha \mathrm{X}$-ray source with radiation. Field emission scanning electron microscopy (FESEM, JEOL-7100F) and Energy-Dispersive Spectroscopy (EDS) were used to analyze morphology and element distribution. Transmission electron microscopy (TEM) images were obtained by using JEM-2100F with acceleration voltage $200 \mathrm{kV}$. Fourier transform infrared Spectroscopy (FTIR) measurements were collected by using Nicolet 6700 (Thermo Fisher Scientific Co., USA) IR spectrometer with wavenumber range from 400 to $4000 \mathrm{~cm}^{-1}$. Thermogravimetric analysis (TGA) was conducted with a STA-449C from 30 to $600{ }^{\circ} \mathrm{C}$. The Tristar II 3020 instrument was performed to measure BET specific surface area and pore volume by adsorption of nitrogen at $77 \mathrm{~K}$. The X-ray photoelectron spectroscopy (XPS) analysis was tested by ESCALAB $250 \mathrm{Xi}$ spectrometer. Raman spectroscopy was performed on Renishaw INVIA micro-Raman spectroscopy system.

\subsection{Electrochemical Measurements}

100 mg PLM@LE was pressed into blocks at 6 T pressure and assembled into CR2025 coin cells for Electrochemical impedance spectroscopy (EIS) test. The ionic conductivity of stainless steel (SS)//PLM@LE//SS was measured from $0.1 \mathrm{~Hz}$ to $1000 \mathrm{kHz}$ by AutoLab PGSTAT302N. The electrochemical window of SS//PLM@LE//Na was tested by linear sweep voltammetry (LSV) from 1 to $6 \mathrm{~V}$ at a scan rate of $1 \mathrm{mV} \mathrm{s}^{-1}$. Na//PLM@LE//Na symmetric battery was assembled and tested on the multichannel battery testing system (LAND CT2001A) to monitor Na plating/stripping test. Cathode mixture, PLM@LE flexible membrane and sodium metal anode were assembled into CR2016 coin cell for galvanostatic charge/discharge measurements and rate performance test. 


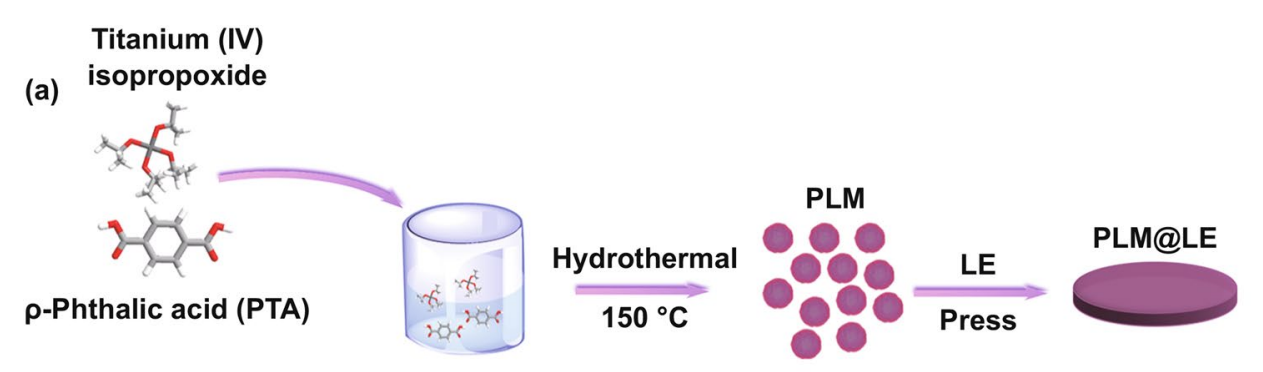

(b)

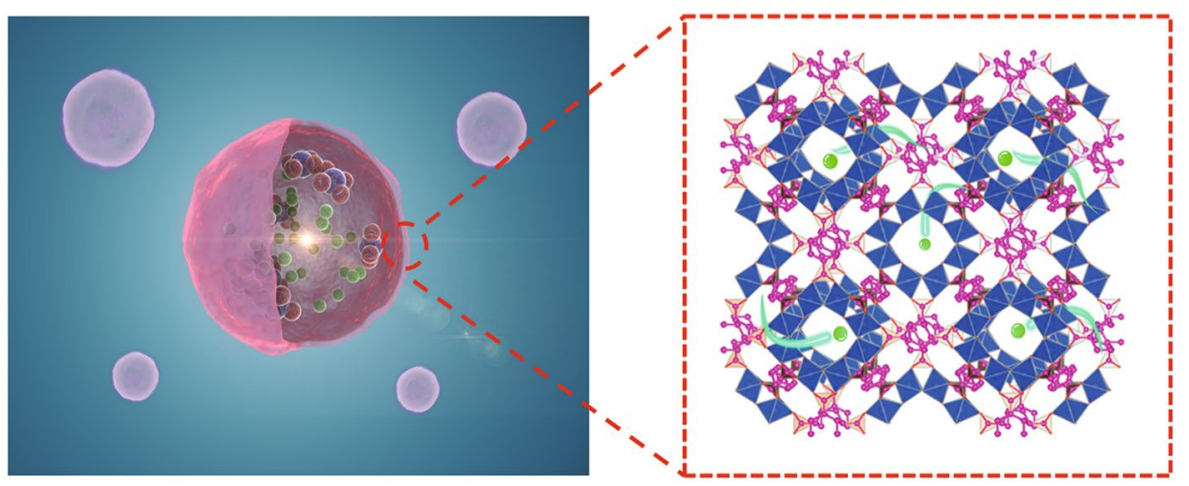

Fig. 1 a Synthetic strategy of PLM@LE. b Top view morphology and crystal structure of PLM (green ball represents sodium ion, and the blue red ball represents other ions or molecules)

\section{Results and Discussion}

\subsection{Morphology and Structure Characterization}

High-performance SLE with pancake-like morphology were synthesized by a simple hydrothermal method (Fig. 1a). The LE we chose is $\mathrm{NaClO}_{4}$-PC with $5 \%$ FEC $\left(\mathrm{NaClO}_{4}\right.$ content is $1 \mathrm{M})$, in which $\mathrm{PC}$ is polycarbonate $\left(\mathrm{C}_{4} \mathrm{H}_{6} \mathrm{O}_{3}\right)$ and FEC is fluoroethylene carbonate $\left(\mathrm{C}_{3} \mathrm{H}_{3} \mathrm{FO}_{3}\right) . \mathrm{NaClO}_{4}$ has high decomposition temperature and high ionic conductivity, while the PC solution has good electrochemical stability and excellent ion transport capacity (FEC is used as an additive to reduce the viscosity of electrolyte) $[9,31]$. As shown in Fig. S1, the longest diameter of PC and FEC molecules is 5.165 and $4.556 \AA$, respectively. PLM was chosen as the MOF material, which is composed of ring clusters with secondary building unit of $\mathrm{Ti}_{8} \mathrm{O}_{8}$ (regular octahedron in blue) and organic ligand $\mathrm{O}_{2} \mathrm{C}-\mathrm{C}_{6} \mathrm{H}_{4}-\mathrm{CO}_{2}$ (magenta). It has two kinds of cages: regular tetrahedron $6.13 \AA$ and octahedron $12.55 \AA$, and its apertures size are mainly distributed in 5-7 $\AA$ [32]. As shown in Fig. 1b, PLM has a pancakelike morphology, which can act as a "host" to hold the LE. In addition, its unique pore sizes can provide unrestricted transport channels for $\mathrm{Na}^{+}$and limit the migration of other ions or molecules.

X-ray diffraction (XRD) peaks of PLM at $6.7^{\circ}, 9.7^{\circ}$, and $11.6^{\circ}$ correspond to the (101), (200), and (211) crystallographic planes (Fig. 2a). The XRD pattern fits with the previous literature [32], indicating that PLM has been successfully synthesized. Scanning electron microscopy (SEM) and transmission electron microscopy (TEM) were used to characterize the morphology of PLM. It was obvious that PLM had a pancake-like morphology with a size of $2 \mu \mathrm{m}$ (Fig. 2b, c). To minimize the occupation of PLM pores by other molecules, PLM powder was dried in an $120^{\circ} \mathrm{C}$ vacuum oven overnight, and the fixed amount of PLM $(0.1 \mathrm{~g})$ was mixed with different contents of LE. The obtained PLM@LE powder was put in the glove box, waiting for the following tests. Similar to the high specific surface area of red blood cells, the BET surface area of PLM was $1112.4 \mathrm{~m}^{2} \mathrm{~g}^{-1}$ and the total pore volume was $0.46 \mathrm{~cm}^{3} \mathrm{~g}^{-1}$ according to the nitrogen adsorption/desorption isothermal tests, confirming the high porosity of PLM host (Fig. 2d). The BET surface area of PLM@LE powder decreased to $2.6 \mathrm{~m}^{2} \mathrm{~g}^{-1}$ indicates that the LE has successfully occupied the channel of PLM (Fig. S2). With the successful implantation of different LE contents, the PLM skeleton did not appear obvious collapse which 

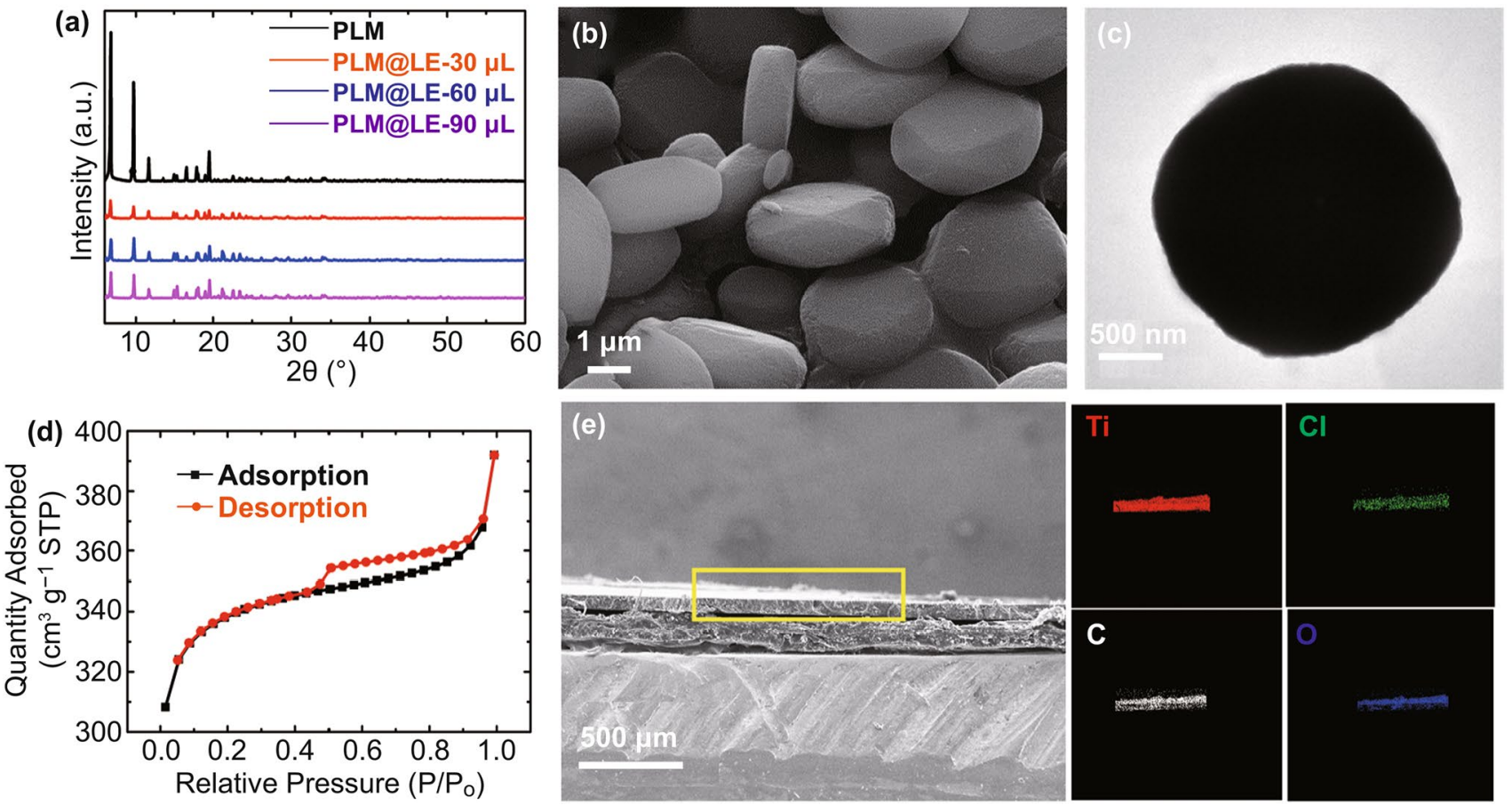

Fig. 2 a XRD patterns of PLM and PLM with different LE contents. b SEM and $\mathbf{c}$ TEM image of PLM. d $\mathrm{N}_{2}$ adsorption/desorption isothermal linear plots of PLM. e Cross-sectional SEM and EDS images of PLM

was proved by XRD patterns (Fig. 2a) and Raman spectra (Fig. S3) of PLM@LE. Although there was no characteristic peak of LE, the intensity of the PLM@LE peaks decreased obviously, which indicated that LE existed in the internal framework of PLM. The SEM images of PLM with different contents LE were shown in Fig. S4. With the increase in LE content, the pancake-like morphology of PLM has no obvious change. In addition, PLM@LE samples with different LE were further analyzed by X photoelectron spectroscopy (XPS) as shown in Fig. S5. It is worth mentioning that the excessive LE $(120 \mu \mathrm{L})$ was difficult to detect by XPS. A strong $\mathrm{Cl}$ peak was detected with the addition of LE, and the $\mathrm{C}$ peak based on PLM had no position shift, which indicates that there was no change of valence electrons with the mix of LE.

In order to explore the advantages of the pancake-like morphology of PLM, a decahedron-like material about $2 \mu \mathrm{m}$ was prepared with different solvent contents, as shown in Fig. S6a, b. XRD peaks at $6.7^{\circ}, 9.7^{\circ}$, and $11.6^{\circ}$ fully demonstrate that the decanedron-like MOF (DLM) was still MIL125 (Fig. S6c). The BET surface area and pore volume of DLM was $1049.9 \mathrm{~m}^{2} \mathrm{~g}^{-1}$ and $0.43 \mathrm{~cm}^{3} \mathrm{~g}^{-1}$, respectively (Fig. S6d). Compared with PLM, the internal pore diameter distribution of DLM was almost the same as PLM, which indicates the consistency of the internal aperture distribution between PLM and DLM (Fig. S6e, f). Furthermore, the specific surface area of DLM decreased, which was caused by the decrease in external specific surface area or/ and missing linkers. To study the influence of structural defects or site impurities of MOF on the electrochemical performance, we added the $\mathrm{NH}_{2}$ functional group to the PLM ( $\mathrm{NH}_{2}$-MIL-125). It is obvious that $\mathrm{NH}_{2}$-MIL-125 still has a pancake-like morphology, and the inset shows that the $\mathrm{NH}_{2}$-MIL-125 is a yellow powder (Fig. S7a). In addition, the XRD peaks maintains a good similar crystal pattern of PLM (Fig. S7b). FTIR spectrum was used to further analyze the structure of $\mathrm{NH}_{2}-\mathrm{MIL}-125$, and it was found that there were two small peaks at 3467 and $3436 \mathrm{~cm}^{-1}$, which represented the contraction vibration peaks of $\mathrm{NH}_{2}$ (Fig. S7c). Subsequently, the high specific surface area of $\mathrm{NH}_{2}$-MIL-125 $\left(1126.9 \mathrm{~m}^{2} \mathrm{~g}^{-1}\right.$ ) was found, indicating that the $\mathrm{NH}_{2}$ had no significant effect on the specific surface area (Fig. S7d).

At the same time, the thermal stability of PLM, PLM@ LE and DLM was tested (Fig. S8). There are two stages of weight loss in PLM. In the first stage, the weight loss is in the range of $80-120{ }^{\circ} \mathrm{C}$, mainly cause by the loss of water 
or solvent molecules in the pores. In the second stage, the weight loss begins at about $300{ }^{\circ} \mathrm{C}$ and ends at about $450{ }^{\circ} \mathrm{C}$. This is due to the collapse of the framework accompanied by the decomposition of organic ligands [32]. The thermal stability of DLM was similar to PLM, indicating that the change of crystallographic planes proportion has little influence on the thermal stability. A sharp decline of PLM@LE occurred between 100 and $200{ }^{\circ} \mathrm{C}$ due to the addition of LE, but the subsequent decomposition trend of high temperature section was same as the PLM skeleton, indicating that LE was almost completely decomposed at $200{ }^{\circ} \mathrm{C}$. Moreover, PLM@LE white powder was pressed under 6 T pressure and kept the pressure for one minute. The PLM was closely packed together with no obvious cracks on the surface (Fig. S9a, b). High structural stability similar to red blood cells, pancake-like morphology reduced the stress on a single PLM, ensuring the high safety of PLM@LE under high pressure. However, owing to the decahedron-like morphology of DLM, the structure of DLM collapsed and broke obviously under the same pressure, leading to the increase in sodium ion transport barrier in DLM (Fig. S9c, d). The cross-sectional SEM and the energy-dispersive spectrometer (EDS) elemental mappings show that the overall thickness of the SLE was about $120 \mu \mathrm{m}$ and the $\mathrm{Cl}$ element was uniformly distributed in the PLM@LE, which implies that the LE remains in the PLM after pressed at $6 \mathrm{~T}$ high pressure (Fig. 2e).

\subsection{Electrochemical Performance and Ion Transport Mechanism}

The content of LE is directly related to the electrochemical properties of PLM@LE. The PLM@LE with different LE were assembled into stainless steel (SS)//PLM@LE//SS to perform electrochemical impedance spectroscopy (EIS) at $20-70{ }^{\circ} \mathrm{C}$. The Arrhenius curves with different LE contents were shown in Fig. 3a. With the LE content increase from 60, 90 to $120 \mu \mathrm{L}$, the activation energy of PLM@LE was $0.199,0.112$, and $0.082 \mathrm{eV}$, respectively. The activation energy indicates the $\mathrm{Na}^{+}$migration ability in PLM organic skeleton. The lower the activation energy value, the easier the $\mathrm{Na}^{+}$transport. But unfortunately, $120 \mu \mathrm{L}$ PLM@LE was no longer a dry-solid powder, which represents that it cannot completely absorb LE (Video S1). Therefore, the optimal ratio (MOF: $\mathrm{LE}=0.1 \mathrm{~g}: 90 \mu \mathrm{L}$ ) was determined and used for the subsequent testing. The EIS curves of different temperatures were shown in Fig. 3b. The ionic conductivity of PLM@LE at 20 and $70{ }^{\circ} \mathrm{C}$ was $6.60 \times 10^{-4}$ and $1.15 \times 10^{-3}$ $\mathrm{S} \mathrm{cm}^{-1}$, respectively. In addition, DLM was compounded with $90 \mu \mathrm{L}$ of LE and assembled into SS//DLM@LE//SS for the same Arrhenius and EIS tests. The activation energy of DLM@LE was $0.135 \mathrm{eV}$, which was higher than $0.112 \mathrm{eV}$ of PLM@LE (Fig. 3c, d). The ionic conductivity of DLM@ LE was $2.56 \times 10^{-4}$ and $3.86 \times 10^{-4} \mathrm{~S} \mathrm{~cm}^{-1}$ at 20 and $70^{\circ} \mathrm{C}$, respectively. The difference between PLM@LE and DLM@ LE was ascribed to the high liquid retention of pancake-like
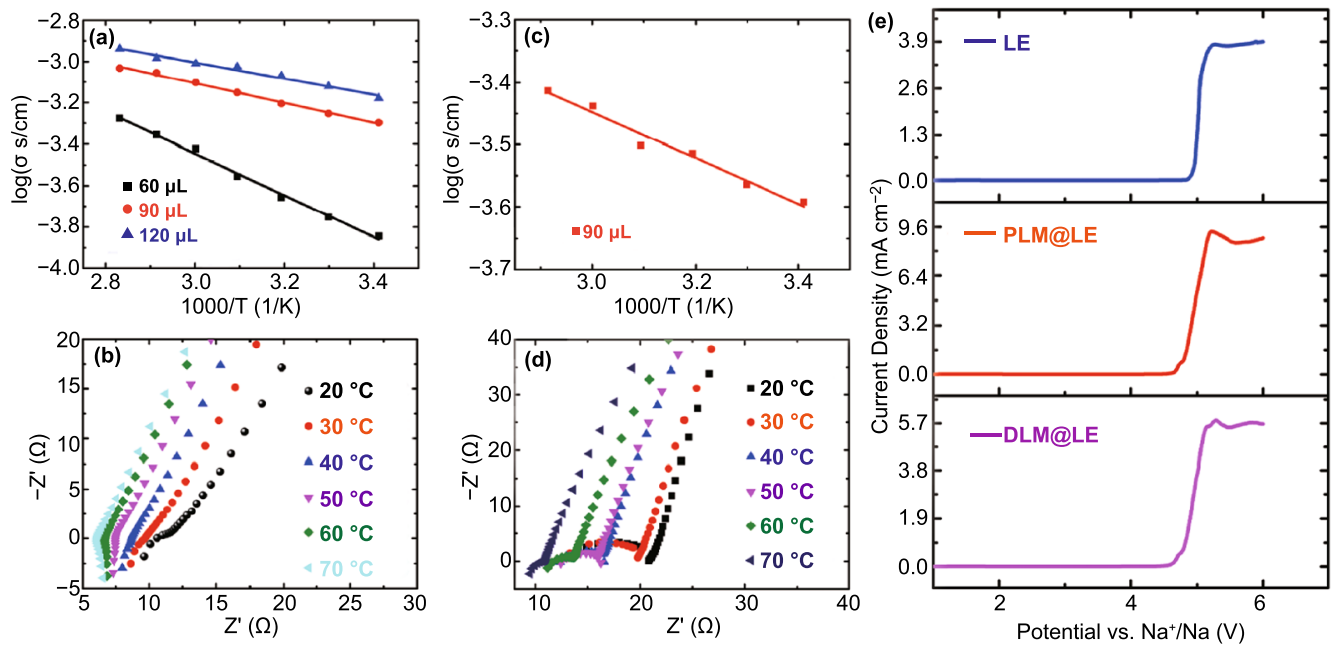

Fig. 3 a Arrhenius plots for the ionic conductivity of PLM@LE with different LE contents. b EIS of PLM@LE (0.1 g: $90 \mu L)$ at temperatures from 20 to $70{ }^{\circ} \mathrm{C}$. c Arrhenius plot and d EIS of DLM@LE $(0.1 \mathrm{~g}: 90 \mu \mathrm{L})$ at different temperatures. e LSV of DLM@LE, LE and PLM@LE under a scan rate of $1 \mathrm{mV} \mathrm{s}^{-1}$ at $23{ }^{\circ} \mathrm{C}$ 
morphology. What' more, the ionic conductivity at room temperature and activation energy of $\mathrm{NH}_{2}$-MIL-125 was $6.08 \times 10^{-4} \mathrm{~S} \mathrm{~cm}^{-1}$ and $0.1167 \mathrm{eV}$, respectively (Fig. S10). These results indicate that the electrochemical properties of the pancake-like MOF after ammoniation are still very similar to PLM@LE, although the internal sites of MOF are changed. Electrochemical windows of PLM@LE and DLM@LE were measured at room temperature by linear sweep voltammetry (LSV), as shown in Fig. 3e. The electrochemical windows of PLM@LE and DLM@LE were stable in the range of $1-4.8 \mathrm{~V}$, indicating the electrochemical stability of MOF. To further demonstrate the electrochemical stability of MOF, CV tests were performed at voltages from - 0.5 to 2 V. It can be clearly seen that CV curves in PLM@ LE can maintain a high degree of consistency after CV cycles, indicating that MOF has a good stability to sodium metal (Fig. S11).

The sodium ion transference number $\left(t_{\mathrm{Na}+}\right)$ was tested by assembling a Na symmetrical cell at room temperature with a constant voltage of $10 \mathrm{mV}$ according to the literature method (Fig. 4a-c) [33]. According to the EIS test, the interface resistance of PLM@LE against sodium was lower than DLM@LE, which matched the previous EIS test. In addition, the $t_{\mathrm{Na}}$ of the initial LE was only 0.16 , indicating the main type of ionic conductance was $\mathrm{ClO}^{-}{ }_{4}$ rather than $\mathrm{Na}^{+}$. The $t_{\mathrm{Na}+}$ of PLM@LE and DLM@LE increased significantly to 0.33 and 0.28 because of the aperture size of MIL-125 host. The size of its pore is about $5 \AA$, which could restrict migration of PC (5.165 ̊) and FEC (4.556 ̊). According to the previous report, $\mathrm{PC}$ molecules and $\mathrm{Na}^{+}$tend to interact with each other near carbonyl oxygen atoms, which increases the diffusion barrier of $\mathrm{Na}^{+}$[34]. More recently, Chang et al. have realized the de-solvated of a lithium-ion electrolyte through an ordered hole in the electrically insulated MOF [35]. Therefore, limiting some of the solvent molecules migration will increase the de-solvated process of $\mathrm{Na}^{+}$and the $t_{\mathrm{Na}+}$. What's more, we provide another possible explanation, because the strong electronegativity of some special functional group leads to an increase in the density of the electron cloud, and there might be a repulsive force against the electron-rich material [36, 37]. Therefore, XPS, FTIR and Raman spectroscopy were used to analyze the existence of special functional groups on the MOF skeleton. High-resolution XPS spectrum of C $1 \mathrm{~s}$ for PLM in Fig. 4d showed that the three peaks at binding energies of $288.67,286.27$, and $284.77 \mathrm{eV}$ corresponded to covalent $\mathrm{O}-\mathrm{C}=\mathrm{O}, \mathrm{C}-\mathrm{O}-\mathrm{C}$, and $\mathrm{C}-\mathrm{C}$ bonds, [38]. In addition, the high-resolution XPS spectra of $\mathrm{O} 1 \mathrm{~s}$ reflected the existence of covalent $\mathrm{C}=\mathrm{O}$ bond at the binding energy of $532.22 \mathrm{eV}$ (Fig. S5) [39]. Moreover, FTIR spectrum further proved the existence of $\mathrm{C}=\mathrm{O}$. As shown in Fig. 4e, the FTIR peaks at 1706 and $1653 \mathrm{~cm}^{-1}$ represent the stretching vibration of
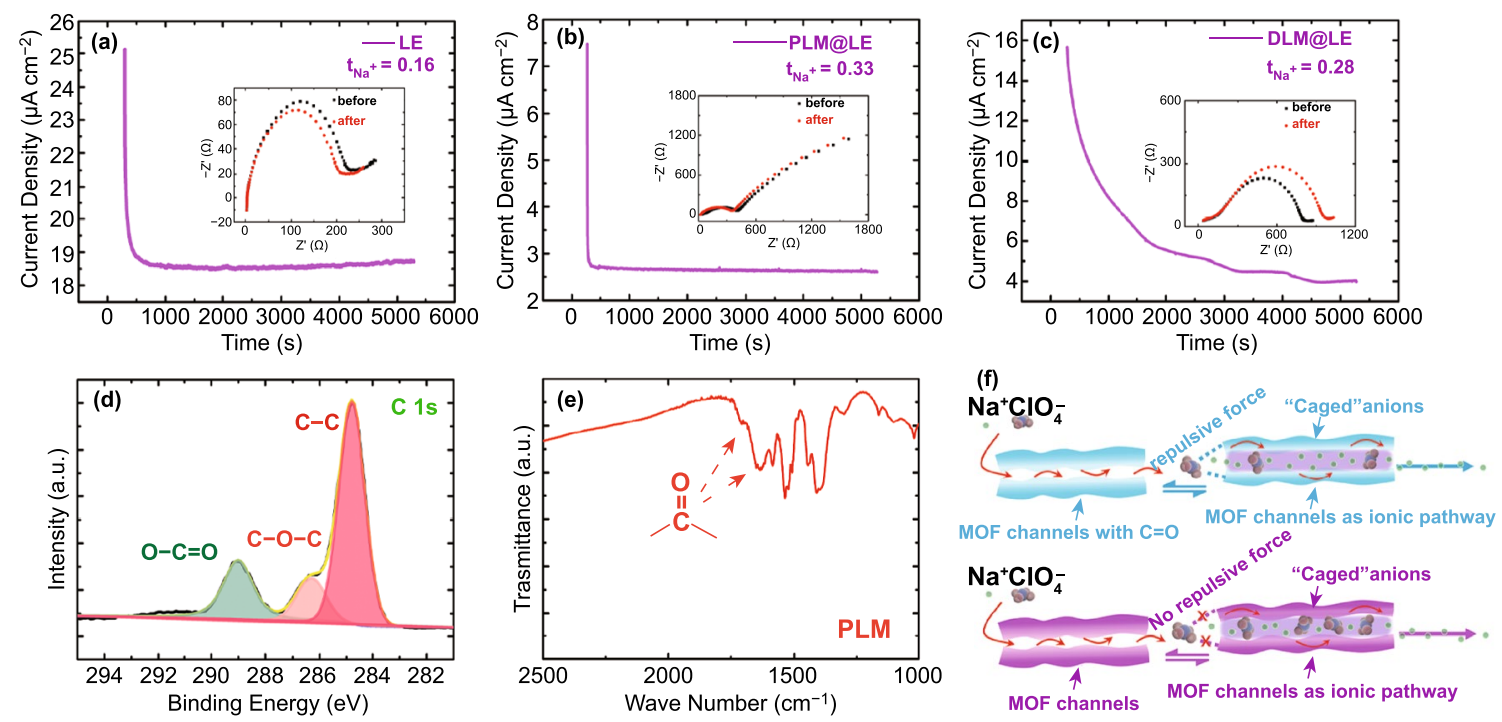

Fig. 4 Current-time profile for a Na//LE//Na, b Na//PLM@LE//Na and c Na//DLM@LE//Na cell. Inset: EIS before and after polarization. d High-resolution XPS spectrum of C $1 \mathrm{~s}$ for PLM. e FTIR spectrum of PLM. f Schematic of the selective $\mathrm{Na}^{+}$ionic transport imposed by the MOF host 
carbonyl function $\mathrm{C}=\mathrm{O}$ [40]. The strong electronegativity of oxygen in the carbonyl group makes carbonyl have a strong nucleophilic property. Strong repulsive force could restrict the migration of anions, which leads to the increase in $t_{\mathrm{Na}+}$ (the mechanism diagram was shown in Fig. 4f). DLM was characterized by FTIR, as shown in Fig. S12a. $\mathrm{C}=\mathrm{O}$ peaks appeared at 1715 and $1628 \mathrm{~cm}^{-1}$, and the overall peak positions corresponded to PLM, indicating the consistency of organic functional groups. Raman spectra in Fig. S12b further demonstrate the existence of $\mathrm{C}=\mathrm{O}$ and the consistency between PLM and DLM. The four strong peaks at 1618, 1435,1146 , and $865 \mathrm{~cm}^{-1}$ correspond to the bending vibrations and stretching of the $\mathrm{C}=\mathrm{O}$ and the $\mathrm{C}=\mathrm{C}, \mathrm{C}-\mathrm{H}$ vibrations on the benzene ring.

\subsection{Interface Analysis and Solid-State Battery Performance}

The direct current (DC) Na plating/stripping experiments were used to measure the impedance of sodium ion migration at the interface and the stability of SLE against sodium metal. Figure 5a shows the voltage-time plots of the $\mathrm{Na} / /$ PLM@LE//Na symmetric cells at different current densities. The polarization voltage of PLM@LE was no more than $\pm 90, \pm 120$, and $\pm 150 \mathrm{mV}$ at $0.2,0.4$, and $0.6 \mathrm{~mA} \mathrm{~cm}^{-2}$, respectively. This low interfacial impedance and excellent interfacial compatibility was ascribed to the wetting effect of LE on the interfaces. The contact between MOF and sodium metal was not completely solid-solid contact, which constructed a fast path for the rapid transport of sodium ions
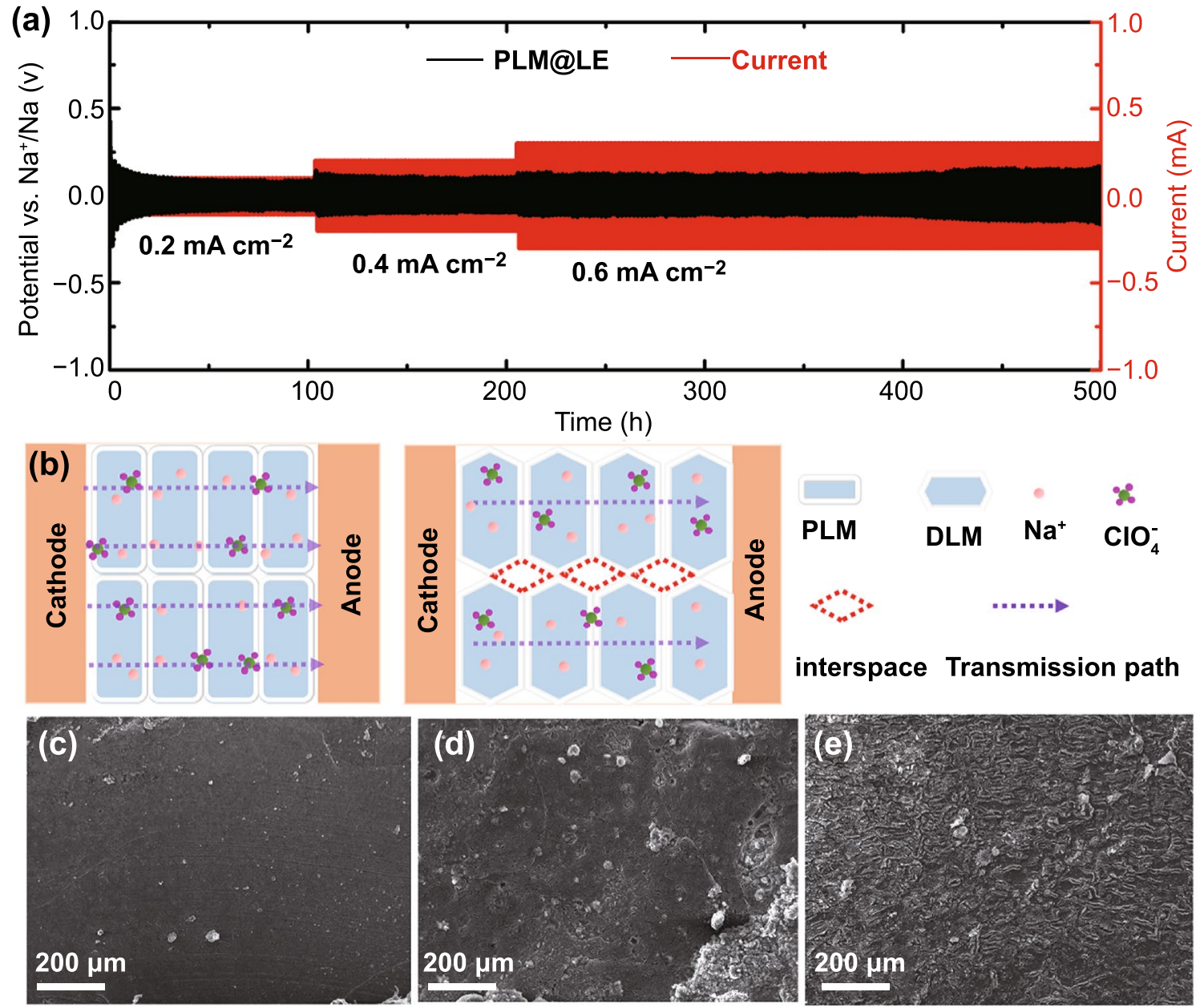

Fig. 5 a Direct current Na plating/stripping of Na//PLM@LE//Na at current densities of 0.2, 0.4 and $0.6 \mathrm{~mA} \mathrm{~cm}{ }^{-2}$. b Schematic of the PLM@ LE and DLM@LE internal interface contact. SEM images of sodium metal $\mathbf{c}$ original, d Na//PLM@LE//Na and e Na//DLM@LE//Na 

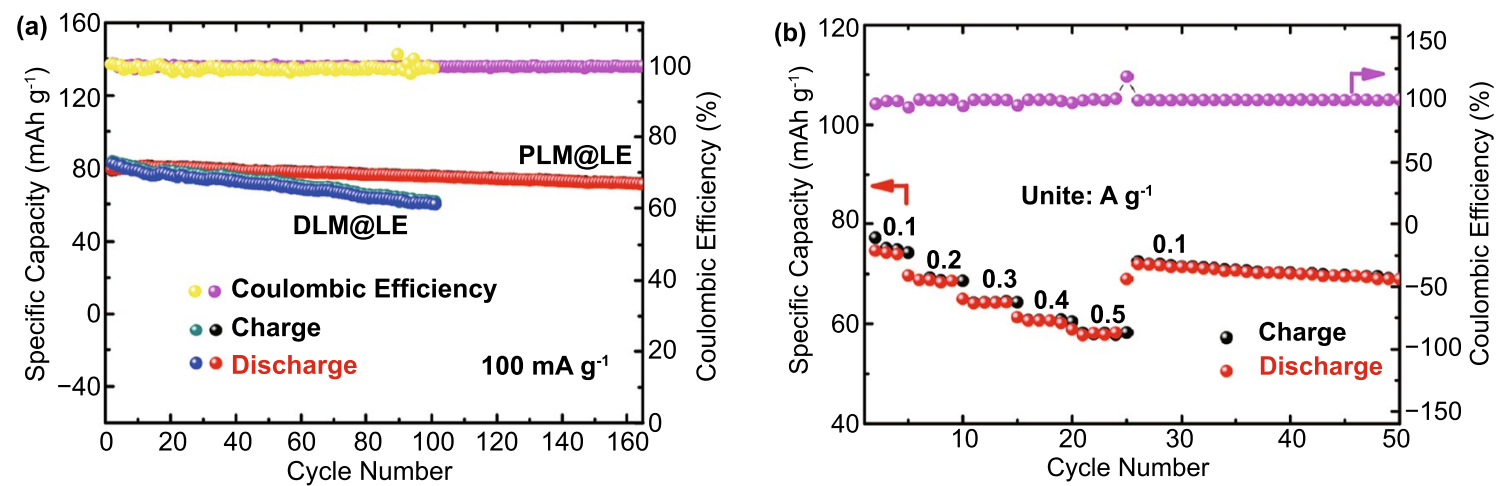

Fig. 6 a Cycling performance of $\mathrm{Na}_{0.44} \mathrm{MnO}_{2} / / \mathrm{PLM} @ \mathrm{LE} / / \mathrm{Na}$ and $\mathrm{Na}_{0.44} \mathrm{MnO}_{2} / / \mathrm{DLM} @ \mathrm{LE} / / \mathrm{Na}$ solid batteries at $100 \mathrm{~mA} \mathrm{~g}^{-1}$. b Rate performance of $\mathrm{Na}_{0.44} \mathrm{MnO}_{2} / / \mathrm{PLM} @ \mathrm{LE} / / \mathrm{Na}$

in interfaces [41]. In addition, PLM@LE can cycle steadily for more than $500 \mathrm{~h}$, representing the interfacial stability between the PLM@LE and sodium metal during the cycle. In addition, the three characteristic peaks of PLM still exist obviously at $6.7^{\circ}, 9.7^{\circ}$, and $11.6^{\circ}$, indicating that PLM has good chemical stability after the cycling process (Fig. S13). The voltage-time plots of the PLM@LE and DLM@LE at $0.4 \mathrm{~mA} \mathrm{~cm}^{-2}$ current density were shown in Fig. S14. It can be seen that the polarization of DLM@LE increases significantly during the cycle, which indicates the poor compatibility with sodium metal. The reason for this phenomenon was shown in Fig. 5b. From the microscopic point of view, the good contact between the smooth PLM leads to a lower sodium ion transport barrier in PLM interfaces. In addition, sodium dendrites tend to grow in the cracks of DLM@LE during the cycling process, resulting in the fragmentation or pulverization of electrolytes. The SEM image of sodium metal before cycling was shown in Fig. 5c, which shows that the surface of sodium metal was very smooth without the appearance of sodium dendrites. Compared with DLM@ LE, the dendrite growth of sodium metal on PLM@LE was inhibited to a greater extent because the sodium dendrite did not grow conveniently in the PLM interstitial space (Fig. 5d, e). Figure S15 represents the inhibition of sodium dendrite in PLM@LE at different current densities, which indicates the electrochemical superiority of PLM@LE.

To further explore the application prospect of PLM@LE, $\mathrm{Na}_{0.44} \mathrm{MnO}_{2} / / \mathrm{PLM} @ \mathrm{LE} / / \mathrm{Na}$ full battery was assembled. The charge/discharge cycling at $100 \mathrm{~mA} \mathrm{~g}^{-1}$ current density was shown in Fig. 6a. It is worth mentioning that the capacity has a standard deviation of $4 \mathrm{mAh} \mathrm{g}^{-1}$. The discharge capacity has a slight increase from 79.4 to $80.9 \mathrm{mAh} \mathrm{g}^{-1}$ in the first
12 cycles. This is because the interface will gradually be completely infiltrated by the LE during the cycling process, leading to an increase in capacity, which was consistent with the DC test results. After 160 cycles, the specific capacity decreased slightly to $71.4 \mathrm{mAh} \mathrm{g}^{-1}$. At the same time, the full battery of DLM@LE showed an initial specific capacity of $83.1 \mathrm{mAh} \mathrm{g}^{-1}$ at a current density of $100 \mathrm{~mA} \mathrm{~g}^{-1}$, but the capacity continued to decline after 100 cycles with a capacity retention rate of $72.9 \%$. Subsequently, rate performance of PLM@LE was measured at different current densities of 100, 200, 300, 400, 500, and $100 \mathrm{~mA} \mathrm{~g}^{-1}$, and the discharge capacities were $74.3,68.8,64.3,60.6,58.1$, and $72 \mathrm{mAh} \mathrm{g}^{-1}$ (Fig. 6b), which fully demonstrated that PLM@LE has a high structural stability and a good recoverable cycle. What's more, the cross-sectional SEM image of $\mathrm{Na}_{0.44} \mathrm{MnO}_{2} / / \mathrm{PLM} @$ LE (Fig. S16) and EIS tests before and after the cycling (Fig. S17) can provide further evidence of good contact between PLM@LE and the electrode. The findings showed that PLM@LE possesses high ionic conductivity, excellent interfacial compatibility and simple preparation technology. All the above results indicate that PLM@LE has a high application value in high-performance SIBs.

\section{Conclusion}

In summary, we constructed a novel SLE based on a MOF with pancake-like morphology. This special morphology of MOF makes PLM have a higher specific surface area to accommodate more LE and block the transport of anions, so that PLM@LE has a high ionic conductivity $\left(6.60 \times 10^{-4} \mathrm{~S}\right.$ $\mathrm{cm}^{-1}$ ) and higher sodium ion transference number (0.33). In addition, by analyzing the influence of different morphology 
of SLE, it was found that PLM@LE had better interface contact, which inhibited the growth of sodium dendrite to a great extent and shown good sodium metal compatibility over $500 \mathrm{~h}$. What's more, characterization of $\mathrm{C}=\mathrm{O}$ functional groups on MOF provides another possible repulsive force explanation for the restriction of anion transport. Finally, after 160 cycles at $100 \mathrm{~mA} \mathrm{~g}^{-1}$, the specific capacity retention rate of the assembled full battery was $88 \%$. This work provides a novel idea for the design of fast ion transfer, high-performance $\mathrm{Na}^{+}$SLE.

Acknowledgements This work was supported by the National Natural Science Foundation of China (51802239), the National Key Research and Development Program of China (2020YFA0 715000, 2019YFA0704902), Foshan Xianhu Laboratory of the Advanced Energy Science and Technology Guangdong Laboratory (XHT2020-005, XHT2020-003), the Natural Science Foundation of Hubei Province (2019CFA001), the Fundamental Research Funds for the Central Universities (2020III011GX, 2020IVB057, 2019IVB054, 2019III062JL), and National Innovation and Entrepreneurship Training Program for College Students (202010497080).

Author's Contributions G. Zhang and J. Shu contributed equally to this work. L. Xu and L. Mai were in charge of this scientific research project, and the leaders of actual coordination of contributions. G. Zhang and J. Shu performed all of the experiments and the data analyses as well as wrote the manuscript. X.Y. Cai, W.Y. Zou, L.L. Du, and S. Hu contributed to the conception of the study and revised the manuscript.

Open Access This article is licensed under a Creative Commons Attribution 4.0 International License, which permits use, sharing, adaptation, distribution and reproduction in any medium or format, as long as you give appropriate credit to the original author(s) and the source, provide a link to the Creative Commons licence, and indicate if changes were made. The images or other third party material in this article are included in the article's Creative Commons licence, unless indicated otherwise in a credit line to the material. If material is not included in the article's Creative Commons licence and your intended use is not permitted by statutory regulation or exceeds the permitted use, you will need to obtain permission directly from the copyright holder. To view a copy of this licence, visit http://creativecommons.org/licenses/by/4.0/.

Supplementary Information The online version contains supplementary material available at https://doi.org/10.1007/ s40820-021-00628-0.

\section{References}

1. A. Sternberg, A. Bardow, Power-to-what? Environmental assessment of energy storage systems. Energy Environ. Sci. 8(2), 389-400 (2015). https://doi.org/10.1039/c4ee03051f

2. H.S. Chen, T.N. Cong, W. Yang, C.Q. Tan, Y.L. Li et al., Progress in electrical energy storage system: a critical review. Progr. Nat. Sci. 19(3), 291-312 (2009). https://doi.org/10. 1016/j.pnsc.2008.07.014

3. M.D. Slater, D. Kim, E. Lee, C.S. Johnson, Sodium-ion batteries. Adv. Funct. Mater. 23(8), 947-958 (2013). https://doi. org/10.1002/adfm.201200691

4. H.S. Li, L.L. Peng, Y. Zhu, D.H. Chen, X.G. Zhang et al., An advanced high-energy sodium ion full battery based on nanostructured $\mathrm{Na}_{2} \mathrm{Ti}_{3} \mathrm{O}_{7} / \mathrm{VOPO}_{4}$ layered materials. Energy Environ. Sci. 9(11), 3399-3405 (2016). https://doi.org/10. 1039/c6ee00794e

5. B. Zhang, G. Rousse, D. Foix, R. Dugas, D.A.D. Corte et al., Microsized Sn as advanced anodes in glyme-based electrolyte for Na-ion batteries. Adv. Mater. 28(44), 9824-9830 (2016). https://doi.org/10.1002/adma.201603212

6. X.Q. Ma, L. Zou, W.X. Zhao, Tailoring hollow microflowershaped $\mathrm{CoSe}_{2}$ anodes in sodium ion batteries with high cycling stability. Chem. Commun. 54(74), 10507-10510 (2018). https://doi.org/10.1039/c8cc04426k

7. G.L. Woods, K.L. White, D.K. Vanderwall, G.P. Li, K.I. Aston et al., A mule cloned from fetal cells by nuclear transfer. Science 301(5636), 1063 (2003). https://doi.org/10.1126/science. 1086743

8. H.-D. Nguyen, G.-T. Kim, J.L. Shi, E. Paillard, P. Judeinstein et al., Nanostructured multi-block copolymer single-ion conductors for safer high-performance lithium batteries. Energy Environ. Sci. 11(11), 3298-3309 (2018). https://doi.org/10. 1039/c8ee02093k

9. Y.X. Huang, L.Z. Zhao, L. Li, M. Xie, F. Wu et al., Electrolytes and electrolyte/electrode interfaces in sodium-ion batteries: from scientific research to practical application. Adv. Mater. 31(21), 1808393 (2019). https://doi.org/10.1002/adma. 201808393

10. Y. Lu, L. Li, Q. Zhang, Z.Q. Niu, J. Chen, Electrolyte and interface engineering for solid-state sodium batteries. Joule 2(9), 1747-1770 (2018). https://doi.org/10.1016/j.joule.2018. 07.028

11. L. Xu, S. Tang, Y. Cheng, K.Y. Wang, J.Y. Liang et al., Interfaces in solid-state lithium batteries. Joule 2(10), 1991-2015 (2018). https://doi.org/10.1016/j.joule.2018.07.009

12. W.R. Hou, X.W. Guo, X.Y. Shen, K. Amine, H.J. Yu et al., Solid electrolytes and interfaces in all-solid-state sodium batteries: progress and perspective. Nano Energy 52, 279-291 (2018). https://doi.org/10.1016/j.nanoen.2018.07.036

13. L. Porcarelli, A.S. Shaplov, F. Bella, J.R. Nair, D. Mecerreyes et al., Single-ion conducting polymer electrolytes for lithium metal polymer batteries that operate at ambient temperature. ACS Energy Lett. 1(4), 678-682 (2016). https://doi.org/10. 1021/acsenergylett.6b00216 
14. H.C. Gao, L.G. Xue, S. Xin, K. Park, J.B. Goodenough, A plastic-crystal electrolyte interphase for all-solid-state sodium batteries. Angew. Chem. Int. Ed. 56(20), 5541-5545 (2017). https://doi.org/10.1002/anie.201702003

15. F. Lv, Z.Y. Wang, L.Y. Shi, J.F. Zhu, K. Edström et al., Challenges and development of composite solid-state electrolytes for high-performance lithium ion batteries. J. Power Sour. 441, 227175 (2019). https://doi.org/10.1016/j.jpowsour.2019. 227175

16. H.L. Li, M. Eddaoudi, M. O'Keeffe, O.M. Yaghi, Design and synthesis of an exceptionally stable and highly porous metalorganic framework. Nat. Energy 402(6759), 276-279 (1999). https://doi.org/10.1038/46248

17. H.-C. Zhou, J.R. Long, O.M. Yaghi, Introduction to metalorganic frameworks. Chem. Rev. 112(2), 673-674 (2012). https://doi.org/10.1021/cr300014x

18. M. Li, D. Li, M. O’Keeffe, O.M. Yaghi, Topological analysis of metal-organic frameworks with polytopic linkers and/or multiple building units and the minimal transitivity principle. Chem. Rev. 114(2), 1343-1370 (2014). https://doi.org/10. $1021 / \mathrm{cr} 400392 \mathrm{k}$

19. W.G. Lu, Z.W. Wei, Z.-Y. Gu, T.-F. Liu, J. Park et al., Tuning the structure and function of metal-organic frameworks via linker design. Chem. Soc. Rev. 43(16), 5561-5593 (2014). https://doi.org/10.1039/c4cs00003j

20. R. Banerjee, A. Phan, B. Wang, C. Knobler, H. Furukawa et al., High-throughput synthesis of zeolitic imidazolate frameworks and application to $\mathrm{CO}_{2}$ capture. Science 319(5865), 939-943 (2008). https://doi.org/10.1126/science.1152516

21. Z.Q. Wang, R. Tan, H.B. Wang, L.Y. Yang, J.T. Hu et al., A metal-organic-framework-based electrolyte with nanowetted interfaces for high-energy-density solid-state lithium battery. Adv. Mater. 30(2), 1704436 (2018). https://doi.org/10.1002/ adma.201704436

22. Z.Q. Wang, J.T. Hu, L. Han, Z.J. Wang, H.B. Wang et al., A MOF-based single-ion $\mathrm{Zn}^{2+}$ solid electrolyte leading to dendrite-free rechargeable Zn batteries. Nano Energy 56, 92-99 (2019). https://doi.org/10.1016/j.nanoen.2018.11.038

23. S. Ma, L. Shen, Q. Liu, W. Shi, C. Zhang et al., Class of solidlike electrolytes for rechargeable batteries based on metalorganic frameworks infiltrated with liquid electrolytes. ACS Appl. Mater. Interfaces 12(39), 43824-43832 (2020). https:// doi.org/10.1021/acsami.0c13437

24. S.S. Park, Y. Tulchinsky, M. Dinca, Single-ion $\mathrm{Li}^{+}, \mathrm{Na}^{+}$, and $\mathrm{Mg}^{2+}$ solid electrolytes supported by a mesoporous anionic $\mathrm{Cu}$-azolate metal-organic framework. J. Am. Chem. Soc. 139(38), 13260-13263 (2017). https://doi.org/10.1021/jacs. $7 \mathrm{~b} 06197$

25. L. Shen, H.B. Wu, F. Liu, J.L. Brosmer, G.R. Shen et al., Creating lithium-ion electrolytes with biomimetic ionic channels in metal-organic frameworks. Adv. Mater. 30(23), e1707476 (2018). https://doi.org/10.1002/adma.201707476

26. Jo.E.C.J. Li, Q.Q. Liu, L.N. Gao, Y.F. Xu, X.Q. Kong et al., Quasi-solid electrolyte membranes with percolated metalorganic frameworks for practical lithium-metal batteries. J.
Energy Chem. 52, 354-360 (2020). https://doi.org/10.1016/j. jechem.2020.04.013

27. Y.F. Xu, L.N. Gao, L. Shen, Q.Q. Liu, Y.Y. Zhu et al., Iontransport-rectifying layer enables Li-metal batteries with high energy density. Matter 3(5), 1685-1700 (2020). https://doi. org/10.1016/j.matt.2020.08.011

28. Q.X. Mu, H. Wang, X.Y. Gu, Z.R. Stephen, C. Yen et al., Biconcave carbon nanodisks for enhanced drug accumulation and chemo-photothermal tumor therapy. Adv. Healthc. Mater. 8(8), 1801505 (2019). https://doi.org/10.1002/adhm. 201801505

29. C.M. Yu, D.P. Qian, X. Huang, F.F. Han, N. Bao et al., Construction of biconcave hemoglobin-based microcapsules and electrochemical evaluation for its ability of oxygen carry. Sens. Actuators B: Chem. 256, 217-225 (2018). https://doi. org/10.1016/j.snb.2017.09.166

30. C.-H. Wang, C.-H. Yang, J.-K. Chang, Suitability of ionic liquid electrolytes for room-temperature sodium-ion battery applications. Chem. Commun. 52(72), 10890 (2016). https:// doi.org/10.1039/c6cc04625h

31. A. Ponrouch, A.R. Goñi, P.M. Rosa, High capacity hard carbon anodes for sodium ion batteries in additive free electrolyte. Electrochem. Commun. 27, 85-88 (2013). https://doi.org/ 10.1016/j.elecom.2012.10.038

32. M. Dan-Hardi, C. Serre, T. Frot, L. Rozes, G. Maurin et al., A new photoactive crystalline highly porous titanium(IV) dicarboxylate. J. Am. Chem. Soc. 131(31), 10857-10859 (2009). https://doi.org/10.1021/ja903726m

33. J. Evans, C.A. Vincent, P.G. Bruce, Electrochemical measurement of transference numbers in polymer electrolytes. Polymer 28(13), 2324-2328 (1987). https://doi.org/10.1016/ 0032-3861(87)90394-6

34. M. Shakourian-Fard, G. Kamath, K. Smith, H. Xiong, S.K.R.S. Sankaranarayanan, Trends in Na-ion solvation with alkyl-carbonate electrolytes for sodium-ion batteries: insights from first principles calculations. J. Phys. Chem. C 119(40), 22747-22759 (2015). https://doi.org/10.1021/acs.jpcc.5b047 06

35. Z. Chang, Y. Qiao, H. Deng, H.J. Yang, P. He et al., A liquid electrolyte with de-solvated lithium ions for lithium-metal battery. Joule 4(8), 1776-1789 (2020). https://doi.org/10.1016/j. joule.2020.06.011

36. C.X. Liu, Y.Z. Ouyang, B. Jia, Z.Q. Zhu, J.B. Shi et al., Leadenhanced gas-phase stability of multiply charged EDTA anions: a combined experimental and theoretical study. J. Mass Spectrom. 47(6), 769-777 (2012). https://doi.org/10.1002/jms. 3014

37. J. Barman, S. Acharya, C.Z. Zhou, S. Chatterjee, A. Engstrom et al., Non-identical electronic characters of the internucleotidic phosphates in RNA modulate the chemical reactivity of the phosphodiester bonds. Org. Biomol. Chem. 4(5), 928-941 (2006). https://doi.org/10.1039/b516733g

38. A.V. Shchukarev, D.V. Korolkov, XPS study of group IA carbonates. Cent. Eur. J. Chem. 2(2), 347-362 (2004). https://doi. org/10.2478/BF02475578 
39. G. Beamson, D. Briggs, High Resolution XPS of Organic Polymers: The Scienta ESCA300 Database, 1st edn. (Whliy, New York, 1992), p. 295

40. C.Y.Y. Tang, Y.-N. Kwon, J.O. Leckie, Effect of membrane chemistry and coating layer on physiochemical properties of thin film composite polyamide RO and NF membranes.
Desalination 242(1), 149-167 (2009). https://doi.org/10. 1016/j.desal.2008.04

41. Z.Q. Wang, Z.J. Wang, L.Y. Yang, H.B. Wang, Y.L. Song et al., Boosting interfacial $\mathrm{Li}^{+}$transport with a MOF-based ionic conductor for solid-state batteries. Nano Energy 49, 580-587 (2018). https://doi.org/10.1016/j.nanoen.2018.04.076 